\title{
Treatment of invasive cervical resorption leading to an extensive crown destruction: A case report
}

\author{
Elçin Yüzgüleç, ${ }^{1}$ (I) Lale Rizeli, ${ }^{2}$ (i) Ayse Zeynep Zengin, ${ }^{2}$ (I) Cangül Keskin ${ }^{1}$ \\ 'Department of Endodontics, Ondokuz Mayıs University Faculty of Dentistry, Samsun, Turkey \\ ${ }^{2}$ Department of Dentomaxillofacial Radiology, Ondokuz Mayıs University Faculty of Dentistry, Samsun, Turkey
}

Invasive cervical resorption (ICR) is a destructive and progressive resorption type with an uncertain etiology. Cone-beam computed tomography is of great importance for reaching a definitive diagnosis. Once diagnosed, a multistage treatment is required to eliminate the pathology. Removal of excessively vascularized granulation tissue is considered one of the most important steps. Trichloroacetic acid at a concentration of $90 \%$ is the most commonly used chemical agent for this purpose. Furthermore, the granulation tissue that becomes avascular upon application of the acid is removed using bur or hand tools. In this case report, we present root canal treatment using trichloroacetic acid (Sigma-Aldrich, MO, US) at a concentration of $90 \%$ and the restoration with intracanal fiber post of ICR tooth with excessive crown destruction.

Keywords: Internal resorption, trichloroacetic acid, upper incisors.

\section{Introduction}

Root resorption occurs in tooth hard tissues because of odontoclastic reactions and can occur internally or externally depending on the location of the tissue from which it originates and its relationship with the root surface (1). The apical and cervical regions are the most common areas affected by external root resorption (2). External root resorption is also classified into surface resorption, external inflammatory resorption, external cervical resorption (ECR), external replacement resorption, and transient apical resorption $(3,4)$. Heithersay defined ECR as invasive cervical resorption (ICR) because of its invasive and aggressive course (5). Although ICR is rare, the etiology behind it is unclear. Trauma, history of orthodontic treatment, intracoronal bleaching, and surgical procedures have been reported as predisposing factors (3).

Maxillary central incisors constitute $30.4 \%$ of the reported ICR cases (6). ICR is usually asymptomatic and detected incidentally on routine radiographic examination (7). In advanced cases, patients can notice pink discoloration in the cervical region of the tooth depending on the position, size, and thickness of the tissue on the $\operatorname{ICR}(7,8)$. This discoloration results from the highly vascular granulation tissue under resorbed dentin and enamel (1). If the pulpal infection develops, pulpitis symptoms may begin to appear. ICR can be misdiagnosed as "pink spot" because of the pinkish color in the tooth crown (4). Before preceding the treatment, granulation tissue should be removed to prevent resorption progression. Mechanical and chemi-

Cite this article as: Yüzgüleç E, Rizeli L, Zengin AZ, Keskin C. Treatment of invasive cervical resorption leading to an

extensive crown destruction: A case report. Turk Endod J 2021;6:61-66.

Correspondence: Cangül Keskin. Department of Endodontics, Ondokuz Mayıs University

Faculty of Dentistry, Samsun, Turkey.

Tel: +90 362 - 3121919 e-mail: canglkarabulut@gmail.com

Submitted: April 13, 2021 Accepted: May 28, 2021

(C2021 Turkish Endodontic Society 
cal debridement techniques are suggested to eliminate granulation tissue in the resorption area. While mechanical debridement includes the curettage of the tissue, chemical debridement requires the use of acidic solutions (7).

In this case report, we present endodontic treatment of ICR using high concentration trichloroacetic acid (SigmaAldrich, MO, US) and restoration with fiber post in the case of ICR causing excessive crown destruction.

\section{Case Report}

A healthy 19-year-old male patient presented to Oral and Maxillofacial Radiology Clinic, Faculty of Dentistry, Ondokuz Mayis University, with an aesthetic complaint related to his anterior tooth. The clinical examination revealed granulation tissue that completely covered the cervical half of the vestibular surface of tooth no. 21 and caused partial destruction of the enamel tissue (Fig. 1). The color of the coronal half of the crown was observed to turn pink because of this large granulation tissue and there were no caries in the tooth. As the isolation of the tooth was not possible due to tissue loss in the cervical region, the electrical pulp test could not be performed. While the tooth was sensitive to percussion, second-degree mobility was detected according to Miller. The periodontal examination revealed inflammation related to the buccal, mesial, and distal gingiva and a periodontal pocket in the buccal gingival groove. When evaluated in terms of occlusal trauma, the presence of premature contact was detected on the palatal surface of tooth no. 21 .

Furthermore, a heterogeneous radiolucent area extending from the cervical part of the crown of tooth no. 21 to the root with well-circumscribed borders in the coronal part and irregular borders in the cervical part, superposed on the pulp chamber, was detected on the periapical radiograph. A slight widening in the periodontal ligament space in the apical region was also detected (Fig. 2). The images were acquired by a bisecting angle technique using phos-

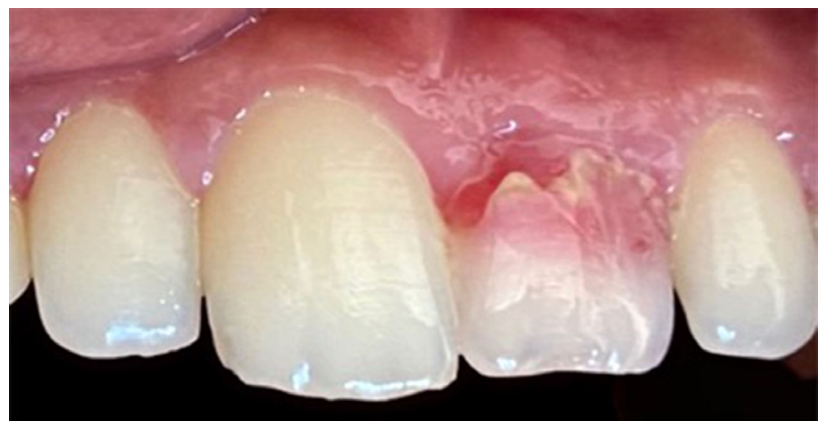

Fig. 1. Intraoral photograph of the patient showing diffuse irregular hard tissue destruction in the resorption area and granulation tissue causing a "pink spot" appearance (A black layer has been added to the background of the image [Adobe Photoshop CS6, Adobe Systems, San Jose, CA, USA]).

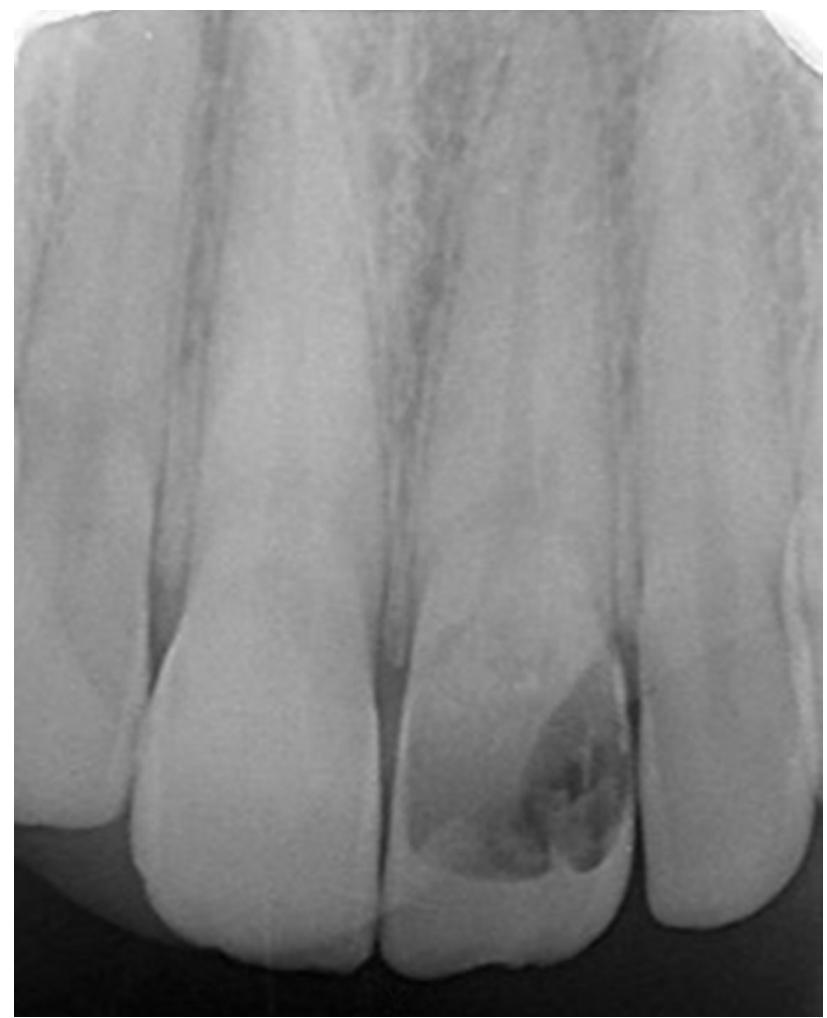

Fig. 2. In the periapical radiograph taken from the patient, a heterogeneous radiolucent area extending from the $2 / 3$ of the cervical part of the crown of tooth no. 21 to the root, with good borders in the coronal part, but irregular and indeterminate borders in the apical part, superposed on the pulp chamber, was observed.

phor plates (VistaScan, Dürr Dental, Bietigheim-Bissingen, Germany) and a dental X-ray device (Sirona Heliodent Plus, Sirona Dental Systems, Bensheim, Germany) with $60 \mathrm{kVp}$ and $7 \mathrm{~mA}$ parameters and scanned using a scanner (Dürr Dental, Bietigheim-Bissingen, Germany). The cone-beam computed tomography (CBCT) images (GALILEOS Comfort Plus, Sirona Dental Systems, Bensheim, Germany; $98 \mathrm{kV}, 15-30 \mathrm{~mA}$, large field of view) showed extension to the mesial and distal surfaces of the crown causing destruction to the vestibular surface, cervical $2 / 3$ of the root. A well-demarcated, heterogeneous hypodense lesion, extending to the middle third of the root, was evident (Fig. 3).

The patient was referred to the endodontics clinic with a prediagnosis of external resorption. The CBCT image taken from the patient showed that there was a radiolucent area compatible with Heithersay (8) type 3 and Patel et al. (9) type 2 cervical resorption. Based on examinations and evaluations, the case was diagnosed as external cervical root resorption. The patient was informed about the diagnosis, treatment options, and possible results. Then, local infiltration anesthesia was applied (Ultracaine D-S Forte $40 \mathrm{mg} / \mathrm{ml}$ articaine $\mathrm{HCl}+12 \mu \mathrm{g} / \mathrm{ml}$ epinephrine 

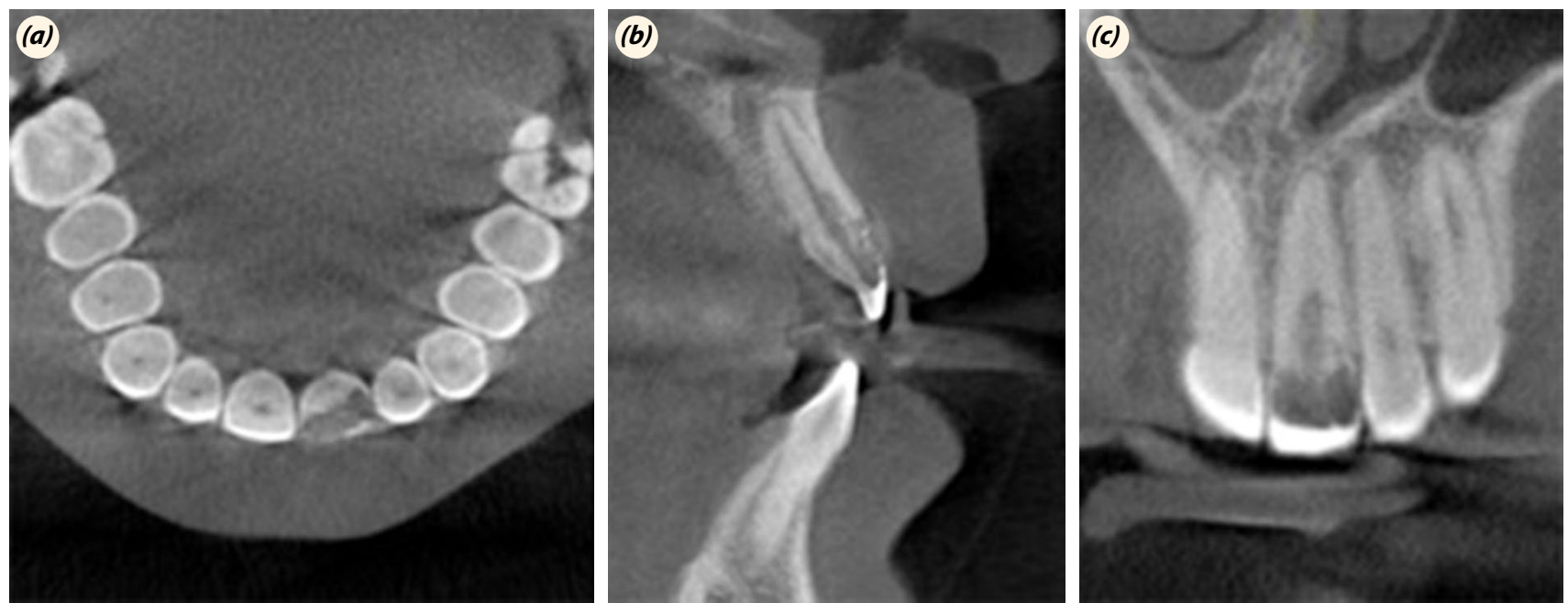

Fig. 3. Cone-beam computed tomography image. (a) The axial section shows a hypodense lesion extending to the mesial and distal surfaces of the crown of the tooth and causing destruction on the vestibular surface. (b) The cross-sectional view shows a heterogeneous hypodense lesion extending to the middle third of the root that includes $2 / 3$ of the cervical part of the crown of the tooth, causing buccal cortical bone destruction. (c) The coronal section shows that a heterogeneous hypodense lesion with a well-demarcated apical is observed in the crown, which extends from two-thirds of the crown to the middle trio of the root. When the images were evaluated, it was found to be compatible with Heithersay type 3 resorption.

$\mathrm{HCl}$ ) after obtaining the patient's written informed consent. Later, because of the loss of dense material in the cervical region, the tooth was isolated using a rubber dam without using a clamp. During the removal of the granulation tissue in the resorption cavity, it was observed that the crown was fractured and the pulp was exposed. After removing the granulation tissue with a sharp-edged excavator, a cotton pellet soaked in trichloroacetic acid at a concentration of $90 \%$ (Sigma-Aldrich, MO, US) was applied to the cavity for 1 minute. The exposed pulp was extracted after the elimination of the granulation tissue using a barbed broach. After determining the working size using an electronic apex finder (Root ZX, Morita, Tokyo, Japan), the canal was shaped to a final size of $\mathbf{5 0 . 0 6}$ with a ProTaper Next nickel-titanium rotary file system (Dentsply Sirona, Ballaigues, Switzerland) up to file number X5. At each file change, the root canal was washed with $5 \%$ sodium hypochlorite (Werax, İzmir, Turkey). A calcium hydroxide medicament (Calcipast, Cerkamed, Stalowa Wola, Poland) was placed in the dried canal, and the tooth was temporarily restored with light-polymerized glass ionomer cement (GC Fuji II LC, Tokyo, Japan). One week later, the tooth was asymptomatic, and the hyperemic condition of the gingiva had improved. After removing the temporary restoration, the resorption cavity was examined under magnification (Zeiss, Oberkochen, Germany). Calcium hydroxide was removed by sonically activated irrigation (EndoActivator, Sirona Dentsply, Ballaigues, Switzerland) with 17\% EDTA (Werax, Izmir, Turkey). The root canal filling of the root canal that was apical of the resorption area was performed with cold lateral compaction technique using epoxy resin-based root canal sealer (AH Plus, Dentsply DeTrey, Konstanz, Germany) and gutta-percha cones. Furthermore, it was decided to restore the tooth with a fiber post due to the excessive loss of coronal material. The patient was given an appointment for the next day, and the tooth was temporarily restored with glass ionomer cement. The canal filling in the apical

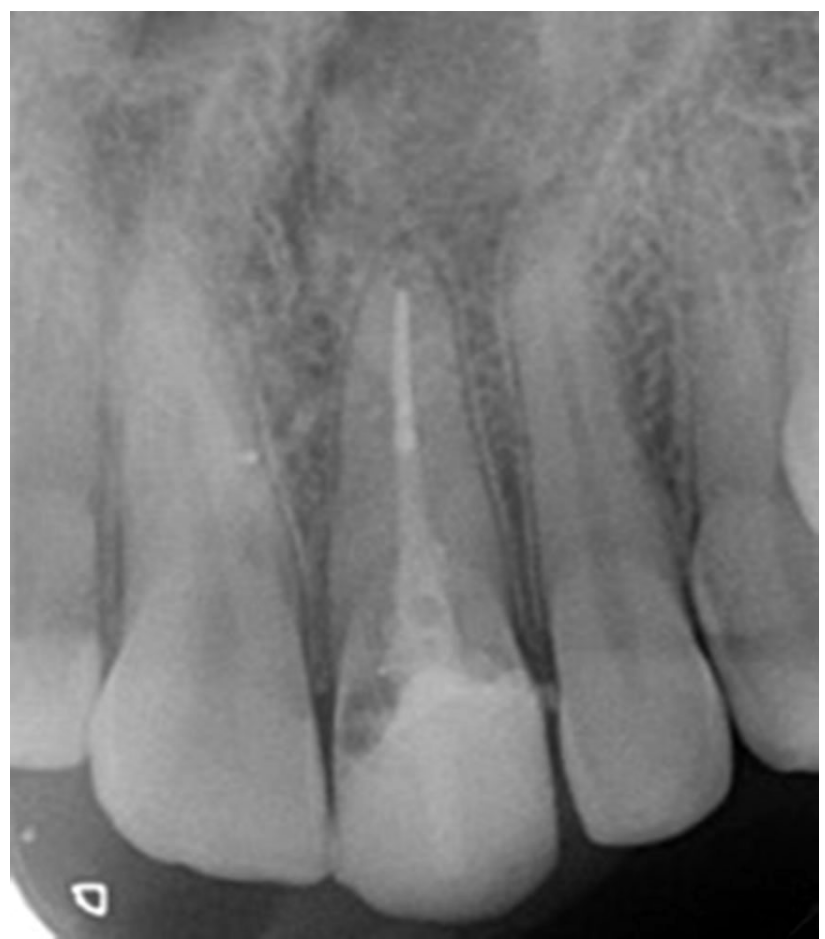

Fig. 4. Periapical radiography of the tooth restored with fiber post after root canal treatment. 


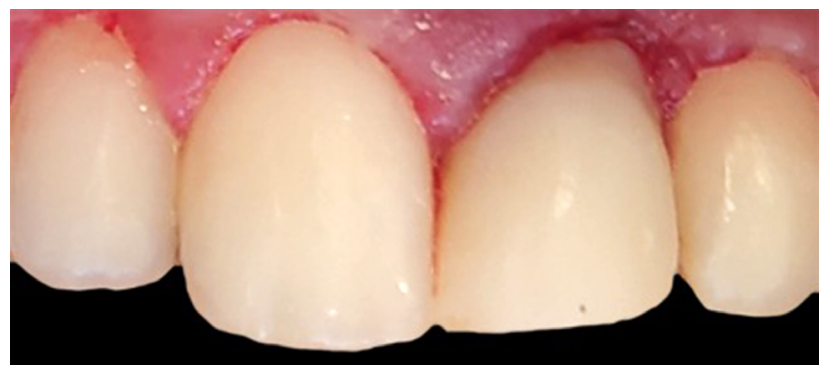

Fig. 5. Intraoral view of the tooth after restoration.

part of the canal up to $5 \mathrm{~mm}$ was removed using the drills of the fiber post system (Cytec Blanco, Hahnenkratt, Germany). In addition, the coronal dentin surface, to which trichloroacetic acid (Sigma-Aldrich, MO, US) was applied previously, was modified with a steel round bur. After applying 37\% orthophosphoric acid (3M ESPE, St. Paul, $\mathrm{MN}$, USA) to the dentin surfaces and drying, the length of the fiber post was adjusted in the canal. The post cavity was bonded (3M Single Bond Universal, 3M ESPE, St. Paul, MN USA) and polymerized for 20 seconds with an LED light device (Woodpecker, Guangxi, China). Dual cure resin cement (RelyX Ultimate, 3M ESPE, MN, USA) was prepared according to the manufacturer's instructions and applied to the canal, and then the fiber post was carefully placed in the root canal and polymerized for $40 \mathrm{sec}-$ onds. The tooth restoration was performed using direct composite resin (Gradia Direct, A2 anterior, GC, Tokyo, Japan). The polishing of the restoration was completed by applying aluminum oxide disks from thick to fine grain (Figs. 4 and 5).

\section{Discussion}

Although ICR cases are rarely detected, early clinical diagnosis is of great importance in tooth prognosis. Moreover, there is a correlation between the occurrence of ICR and devital bleaching, orthodontic treatment, trauma, and surgery history (4). Although the present case does not have a history of dental treatment or trauma, it was determined that the relevant tooth was exposed to occlusal trauma due to premature contact during the clinical examination. Enlarged periodontal ligament space and resorption in the apical region are considered radiological indicators of occlusal trauma (10). In the first session, the premature contact crown fracture detected in the tooth disappeared, and care was taken to protect the tooth from occlusal trauma during the permanent restoration.

ICR lesions can be misdiagnosed as internal resorption, external inflammatory resorption, and extensive cervical caries $(1,11)$. Although differential diagnosis from caries can be easily made by clinical examination, more comprehensive radiological examinations may be required to dis- tinguish it from internal resorption. Periapical radiography is used for radiographic diagnosis. Periapical radiographs can be taken from different angles and applied together with the "Same Lingual Opposite Buccal" rule, in case there is a difficulty in making an internal-external distinction (12). ICR can be seen as unobtrusive or marked radiolucency on periapical radiographs. Lesion appearance can range from well-circumscribed radiolucencies to irregularly-circumscribed radiolucencies (13-15). Radiolucency is superposed on the pulp cavity on radiographs, but even if resorption is widespread, the thin border of the pulp canal could be preserved, and the normal border of the pulp chamber is clearly visible superimposed on the radiolucency (12). If the radiological image of the ICR is superposed on the pulp cavity and the periapical radiograph is not taken from different angles, the ICR may be mistakenly missed as if it was a normal pulp space. The spread and extent of ICR lesions and their proximity to the pulp cavity can also be demonstrated by three-dimensional imaging techniques such as $\mathrm{CBCT}(5,6)$. The buccal and lingual extension and the depth and spread of the lesion can also be shown by CBCT (12).

Internal resorption originates from the vital pulp tissue and causes the shape of the pulp cavity to change as it expands in the lateral direction (8). There is no relationship between ICR and the pulp cavity. Furthermore, the pulp cavity is protected from the resorption lesion by the predentin layer containing pulp resorption inhibitors ( 1 ). In this way, pulp remains vital in ICR lesions like nonperforated internal resorption. In response to pulp that has become necrotic due to trauma, external inflammatory resorption develops and shows a moth-eaten image on the root surface on radiographs (16). CBCT is highly effective and reliable in the diagnosis and evaluation of ICR lesions $(6,17)$. In the present case, CBCT scanning enabled crosssectional imaging of the tooth to determine the limits of resorption.

The priority for the treatment of resorption cases is the elimination of granulation tissue in the resorption area. Trichloroacetic acid is the most commonly used chemical agent used for this purpose $(18,19)$. Its hemostatic effect is achieved by creating coagulation necrosis on the granulation tissue upon direct contact when used at a concentration of $90 \%$ for 1 minute $(11,18)$. Then, bur or hand tools are used to remove the granulation tissue that becomes avascular. Moreover, the solution in liquid form can affect the tissues in areas that cannot be reached using hand tools. Care should be taken during the application of trichloroacetic acid, which is highly caustic, in order to not damage the adjacent soft tissues. Trichloroacetic acid can be used at concentrations as low as $30 \%$ in cases of 
resorption to protect adjacent soft tissues (20). In the literature, it has been reported that the mechanical debridement of resorptive tissue, trichloroacetic acid application, and restoration triad, which is also known as the Heithersay approach, successfully stopped the progression of ICR cases $(11,19)$. It has been reported that the nonsurgical treatment of Heithersay class 3 types of cervical resorption with the application of trichloroacetic acid at a concentration of $90 \%$ is $78 \%$ successful (19). In the present case, it has been observed that the use of trichloroacetic acid at a concentration of $90 \%$ in nonsurgical treatment is very effective and not harmful because the adjacent tissues are protected.

In the present case, dentin surfaces to which trichloroacetic acid (Sigma-Aldrich, MO, US) was applied were modified with steel round. This process, which is done without removing the solid dentin tissue, aims to eliminate the dentin surface demineralized by trichloroacetic acid and to obtain a "renewed" dentin surface for improved adhesion of bonding agents. While the adhesion of a bonding agent to dentin is based on the formation of a hybrid layer by resin infiltration of the collagen matrix released after a superficial demineralization, the adhesion of glass ionomer-based materials depends on ionic bonding to calcium in dentin $(21,22)$. Since no mineral content is left in the part of dentin where trichloroacetic acid is applied, the dentin surface is modified and a refreshed surface is exposed before the application of bonding agent or glass ionomer cement (11).

Fiber posts are commonly used in endodontically-treated teeth with excessive coronal damage (23). In cases where more than two-thirds of the crown is lost, restoration with a post system is recommended (24). The fiber post and direct composite for the permanent restoration provide patient satisfaction due to the preservation of the remaining intact dental tissues and providing aesthetics in a short time. Since the treatment of the current case was performed in the process of the new type of coronavirus pandemic called COVID-19, the restoration of the patient has been completed with fiber post and direct composite to eliminate the functional and aesthetic loss in a minimum number of visits.

\section{Conclusion}

ICR diagnosis and treatment could be difficult due to its complex nature. A good radiological examination and early diagnosis are necessary for the prognosis of the treatment. In the present case, CBCT was used to examine the extension and depth of the lesion, trichloroacetic acid was applied to increase the elimination of resorptive cells due to its rapid hemostatic effect, and finally, a fiber post res- toration was preferred due to the excessive loss of material in the teeth.

Authorship Contributions; Concept: A.Z.Z., C.K.; Design: C.K., E.Y.; Supervision: A.Z.Z., E.Y.; Materials: E.Y, L.R., C.K., A.Z.Z.; Data: E.Y, L.R., C.K.; Analysis: E.Y, L.R., C.K., A.Z.Z.; Literature search: E.Y., L.R.; Writing: E.Y., L.R., C.K., A.Z.Z.; Critical revision: A.Z.Z.

Source of Fundingः None declared.

Conflict of Interest: None declared.

Informed consent: Written informed consent was obtained from patients who participated in this study.

\section{References}

1. Patel S, Kanagasingam S, Pitt Ford T. External cervical resorption: a review. J Endod 2009; 35: 616-25. [CrossRef]

2. White S, Pharoah M. Dental Anomalies. In: Oral radiology principles and interpretation. Missouri: Elsevier Mosby; 2014. p. 604.

3. Patel S, Mavridou AM, Lambrechts P, Saberi N. External cervical resorption-part 1: histopathology, distribution and presentation. Int Endod J 2018; 51: 1205-23. [CrossRef]

4. Atasoy Ulusoy Öİ. External root resorptions and contemporary treatment approaches. ADO Klinik Bilimler Dergisi 2021; 4: 632-7.

5. Heithersay GS. Invasive cervical resorption. Endod Topics 2004; 7: 73-92. [CrossRef]

6. Patel K, Mannocci F, Patel S. The assessment and management of external cervical resorption with periapical radiographs and cone-beam computed tomography: a clinical study. J Endod 2016; 42: 1435-40. [CrossRef]

7. Heithersay GS. Clinical, radiologic, and histopathologic features of invasive cervical resorption. Quintessence Int 1999; 30: 27-37.

8. Heithersay GS. Invasive cervical resorption: an analysis of potential predisposing factors. Quintessence Int 1999; 30: 83-95.

9. Patel S, Foschi F, Mannocci F, Patel K. External cervical resorption: a three-dimensional classification. Int Endod J 2018; 51: 206-14. [CrossRef]

10. Hallmon WW. Occlusal trauma: effect and impact on the periodontium. Ann Periodontol 1999; 4: 102-8. [CrossRef]

11. Schwartz RS, Robbins JW, Rindler E. Management of invasive cervical resorption: observations from three private practices and a report of three cases. J Endod 2010; 36: 1721-30. [CrossRef]

12. Koenig LJ, Tamimi D, Petrikowski CG, Perschbacher SE. Diagnostic Imaging: Oral and Maxillofacial. E-Book. Elsevier Health Sciences; 2017.

13. Yavaş T, Dartar Öztan M, Özen D. Invazive cervical resorption: two case reports. AÜ Diş Hek Fak Derg 2014; 41: 99-106. [CrossRef] 
14. Bakırcı T, Göker Kamalı S, Ağralı ÖB, et al. Management of perforating external cervical root resorption. Eur J Res Dent 2019; 3: 115-8. [CrossRef]

15. Arslan S, Tinaz C, Kayaoğlu G. External cervical root resorption: A case report. Acta Odontol Turcica 2011; 28: $35-39$.

16. Andreasen JO. External root resorption: its implication in dental traumatology, paedodontics, periodontics, orthodontics and endodontics. Int Endod J 1985; 18: 109-18.

17. Patel S, Brown J, Pimentel T, Kelly RD, Abella F, Durack C. Cone beam computed tomography in Endodontics - a review of the literature. Int Endod J 2019; 52: 1138-52.

18. Gulsahi A, Gulsahi K, Ungor M. Invasive cervical resorption: clinical and radiological diagnosis and treatment of 3 cases. Oral Surg Oral Med Oral Pathol Oral Radiol Endod 2007; 103: e65-72. [CrossRef]

19. Heithersay GS. Treatment of invasive cervical resorption: an analysis of results using topical application of trichlor- acetic acid, curettage, and restoration. Quintessence Int 1999; 30: 96-110.

20. Ortega URN, Mejía LR. Use of a lower concentration of trichloroacetic acid to manage invasive cervical resorption: a case report. Endod Pract Today 2019; 13: 71-5.

21. Nakabayashi N, Ashizawa M, Nakamura M. Identification of a resin-dentin hybrid layer in vital human dentin created in vivo: durable bonding to vital dentin. Quintessence Int 1992; 23: 135-41.

22. Lin A, McIntyre NS, Davidson RD. Studies on the adhesion of glass-ionomer cements to dentin. J Dent Res 1992; 71: 1836-41. [CrossRef]

23. Morgano SM, Milot $P$. Clinical success of cast metal posts and cores. J Prosthet Dent 1993; 70: 11-6. [CrossRef]

24. Garoushi SK, Lassila LV, Vallittu PK. Direct composite resin restoration of an anterior tooth: effect of fiber-reinforced composite substructure. Eur J Prosthodont Restor Dent 2007; 15: 61-6. 